\title{
Research on art design and application of indoor environment based on Artificial Intelligence
}

\author{
Hou Yao ${ }^{1}$, Xu Xue-feng ${ }^{1}$ \\ ${ }^{1}$ Changchun University of architecture and Civil Engineering, Changchun,China
}

\begin{abstract}
The application of artificial intelligence in environmental design has entered a stage of rapid development, artificial intelligence in environmental design tools, methods, expression, as well as the expansion of design content, the richness of design language and other aspects have produced a wide range of changes. From the perspective of artificial intelligence in environmental design, this paper discusses the future trend and possible revolutionary changes of artificial intelligence in environmental design. This paper combs the application of artificial intelligence in environmental design, analyzes the design works with wide influence, and discusses the value and significance of artificial intelligence in applied environmental design, as well as its possible future development trend. The application of artificial intelligence in environmental design is a great change from design means to design content. From the interactive ability, virtual ability, to the integration of big data, all are new opportunities and challenges brought by the ability of artificial intelligence to environmental design. In response to such changes, environmental art design practitioners should establish a more systematic, three-dimensional and data-based design activity system and a broader design theory system.
\end{abstract}

\section{Introduction}

The development of new generation information technology is mainly reflected in big data, Internet of things and artificial intelligence, especially artificial intelligence as the leading. Western countries have put forward a new national development strategy which focuses on the development of artificial intelligence. This reflects their emphasis on the manufacturing industry, which is mainly artificial intelligence. The advanced manufacturing in the United States, 4.0 in Germany and the robot revolution in Japan all reflect the ambition of these countries to promote the development of manufacturing industry by science and technology. Since 1950s, artificial intelligence has been developed from the beginning to the high speed, and has penetrated into all walks of life from the research field. In China, the environmental art specialty belongs to the design art under the first level of art. Environmental art plays a great role in the process of economic development in China, whether in landscape architecture or in the construction of indoor and outdoor projects. However, the concept of environmental art is still controversial. In the current discipline, the western countries (such as the United States) have not separated the environmental design majors separately, but they are scattered among the major teaching contents of environmental art, such as landscape architecture, interior design of art academy and urban public art.
The environmental art design in this paper is also a comprehensive expression of design disciplines covering these fields. In recent decades, in the environmental art design, intelligent research work, which is dominated by computer-aided design and machine learning, has been booming, aiming to provide some automatic human tasks, provide personalized knowledge for design experts and provide professional guidance for amateurs. The discussion of this paper also focuses on the development of artificial intelligence in environmental design, focusing on the exploratory research in landscape design and interior design, as well as the thinking and answers brought by relevant research.

\section{Definition, development and current situation of artificial intelligence from the perspective of design}

For the theoretical research of artificial intelligence, the book "artificial intelligence and its application" defines it as: through the establishment of computer model to realize the research of human intelligence behavior, and according to the process of human thinking to carry out creative machine technology. The essence of artificial intelligence is machine intelligence, which can replace human for part of the actual operation. It is a machine, equipment or system that can adapt to the environment independently and complete various tasks according to human thinking consciousness. 
In the field of computer application, artificial intelligence is defined as a new subject based on imitation and expansion of human intelligence, and formed a series of scientific principles, research methods and practical applications. Its purpose is to realize image recognition, speech recognition, human-computer interaction, humancomputer game and autonomous learning in the application of artificial intelligence in intelligent machine or system.

To sum up, although the definition of artificial intelligence in various fields is different, its core significance is to implement human will to achieve user goals. There is no substantial difference in the goal design and value driven orientation of user experience. The key is to simulate human's thinking mode to complete various tasks and goals.

In the mid-1950s, with the concept of artificial intelligence proposed for the first time, countries all over the world have gradually launched the research of artificial intelligence technology, and gradually achieved certain research results, such as checkers program and theorem proving, which can be realized through artificial intelligence technology. This also set off a climax of artificial intelligence research. However, due to the limited ability of machine reasoning, artificial intelligence has gone to the bottom. By the end of 1960s, the emergence of expert system simulating human made artificial intelligence from theoretical research to practical application, and promoted artificial intelligence into a new climax of application development. After entering the 1980 s, due to the continuous expansion of artificial intelligence technology in various fields, the problems of expert system such as narrow application fields, increasing difficulty in acquiring expert knowledge, unable to match with the existing database system gradually appear. In the mid-1990s, with the development of Internet technology, artificial intelligence realized the transformation from single machine to multi-agent learning, which made the artificial intelligence technology more practical. Nowadays, artificial intelligence technology has developed rapidly in different fields, such as scene perception, deep neural network, image recognition and speech recognition. It has realized the effective connection between scientific theory and practical application, such as data mining intelligence technology, unmanned driving intelligence technology and human-computer game. It has broken through many impossibilities in technology application and ushered in a new high of explosive growth Tide.

Based on the application perspective analysis, artificial intelligence can be roughly divided into two stages: special and general. In the application stage of special artificial intelligence, the main body of the system has strong pertinence, the task realization is relatively single, and the modeling process is relatively simple. Through a single breakthrough in various tasks, some data show that artificial intelligence has surpassed human beings in some aspects of single-phase intelligence. For example, in the chess game, human beings are inferior to alpha go, and the "Luban" designed by Alibaba can complete 400 million pieces of online advertising design in one day, far more than human beings. General artificial intelligence is still in the development stage. General artificial intelligence should have the ability of autonomous learning, independent design, rigorous reasoning and scientific judgment, just like human brain, so as to realize "one brain for all purposes". However, the current artificial intelligence technology can not reach this level, only can realize the "shallow intelligence" such as information perception and learning imitation, and the ability of "deep intelligence" such as abstract concepts and reasoning decision-making is still very weak.

Through the analysis of the development process and current situation of artificial intelligence, it can be predicted that it is an inevitable trend to turn from special intelligence to general intelligence, and it is also a forward-looking challenge to realize system development and application.

\section{Artificial intelligence and environment design}

When talking about the relationship between artificial intelligence and landscape design, Yu wrote down several "we embrace artificial intelligence" in passionate tone, and pointed to several application scenarios of AI in landscape design: (1) collection of big data - - efficient and accurate information collection, storage and analysis, which can be used in unprecedented ways to reproduce, perceive and experience landscape; (2) virtual technology - - its powerful operation function and virtual reality technology can enable the computer to display realistic scenes and possible scene changes under complex conditions, and bring the audience experience and aesthetic touch when they are in the scene; (3) liberate the brain and make the design return to creation -- designers can give heavy drawing work to the computer to complete, A large number of logical operations and rational analysis can also be implemented accurately through relevant programming; (4) changing the lifestyle - intelligent life to create more ideal human settlements for designers; (5) hosting dreams - designing and creating beautiful landscape will be an important occupation for the future [3]. The five application scenarios include designers' good thoughts about AI. Relying on the integration of deep learning and big data, artificial intelligence has developed rapidly and has made remarkable achievements in local fields. However, compared with human intelligence, many human activities are simple, even subconscious, which are difficult for machines. This is the famous moravik paradox in the field of artificial intelligence. At present, artificial intelligence, characterized by big data and deep learning, can only solve local specific problems, and can not solve all problems within the scope of human general intelligence, namely "big data and small tasks" mode. The behavior pattern of human world is driven by tasks and created by actions. How to change AI into a "small data big task" way requires several generations of efforts. In the field of environmental art design, the design system of environmental art is a complex and multi-dimensional cross domain integration system, which can be regarded as a large task system. Although the application of artificial intelligence in the local aspect of environmental design 
has been successful, such as big data collection, virtual reality, architectural structure and style analysis, interior furniture furnishing and style analysis, artificial intelligence has a longer way to go compared with the large task system.

\section{Application of artificial intelligence in design}

The word "design" often has different meanings under different premises. As a product, it can be understood as an object being designed, which can be a drawing, a model of a building, a clay slab of a sculpture, a garden building that has been realized or constructed, or other works of art; as a process, design can be understood as a stage of product production, or a presentation means in the process of product formation. Computer aided design software is separated from the design process and product. The difference of the meaning of "design" also shows that the drawing tools represented by computers should be involved in the design process, from representing design information to products, from appearance modeling to visualization of its form, from comprehensive design solutions to evaluating its performance. At present, CAD has two different meanings: one is a tool for drafting and modeling; the other is a design medium. This is just a way for designers to get help in the design process. As a drawing tool and visual means, computer tools have played a huge role in the modern environmental design industry, but they can not ultimately affect the decisionmaking process of designers. On the one hand, the designer's design reasoning comes from his own knowledge reserve and rational and perceptual knowledge of the object, and different types of knowledge reserve determine the designer's decision-making level; on the other hand, the computer-aided means still rely on the huge data sources and the support system constructed by all kinds of information.

The general method to realize artificial intelligence is to establish the operation model of design task. The model is built in two stages. First, cognitive modeling, which is the concept learning stage driven by data, is used to form customization and form a reasonable spatial layout. Since its birth, environmental art design, like its "fellow brothers" architectural design, has established its theory on the basis of mathematics, geometry, the use of materials and the design method of engineering. Human architects have made great progress in these special fields in the design process for thousands of years. With the advent of computers. The engineering means and design methods provided by computer are a fundamental change of modern environmental art, especially architectural design. The application of CAD and CAE replaces the core role of mathematics in environmental design. Second, intelligent system is a tool for optimizing design and fine tuning the newly formed solution. In the specific implementation process, scientists will use different scientific calculation to apply intelligent computing in the field of architecture and interior design. Artificial intelligence is more interesting to try, is in the formation of design style. For example, the styles of different furniture types are compared and studied. The algorithm based on geometric feature vector is carried out by using the big data in the literature. The selected objects are projected into the common k-dimensional space. Through calculation, the application with similar styles is obtained. At present, in the architectural design, landscape design interior design and soft decoration layout, all of them can provide some automatic manual services. The service content should not only include personalized knowledge provided for designers, but also professional guidance services for amateurs. Therefore, in line with other fields, the formation of artificial intelligence also focuses on these aspects.

1) Big data. AI relies on big data to calculate and learn. The more data it has, the stronger its learning ability and the higher its level.

2) The development of software and hardware. The software that can process huge data and the hardware and hardware technology that support the operation of the software are the technical basis for the realization of local artificial intelligence.

3) Deep learning. In the artificial intelligence algorithm which is called neural network and is composed of learning method simulating human brain, deep learning is the most attractive part recently. Nerve cells transmit information to each other through electronic signals. The combination part of information transmission is called synapse. The synaptic connection structure is used for information processing, and the neural network is formed through mathematical modeling. Since the development of convolutional neural network $(\mathrm{CNN})$ and GPU (graphic processing unit) in 2012, the artificial intelligence model with complex semantic features and multidimensional three-dimensional scene construction has been formed [6].

\section{Art creation and artificial intelligence}

In April 2016, a new project was jointly launched by the Royal Moritz Gallery, Rembrandt gallery and Microsoft in the Hague, the Netherlands. A new Rembrandt painting by artificial intelligence has been published. Artificial intelligence scans all the existing Rembrandt paintings, analyzes and graphically processes the theme, strokes and colors of these paintings through in-depth learning, and then creates this work by using $3 \mathrm{D}$ printing technology. This painting has the style of Rembrandt's painting, which is almost unreal. This event in the world, triggered for the use of artificial intelligence for artistic creation of deeper concern, and accelerate the artificial intelligence to explore other areas of artistic creation. In the field of environmental design, due to the complexity of professional properties, similar research has just started. Environmental design covers more complex design elements from macro to micro. It is difficult to integrate and form design results only from personal work analysis and comprehensive calculation of big data. However, from the perspective of development trend, with more and more sufficient sources of big data and enhanced computing power in the future, in the field of environmental art and design, the emergence of works purely designed by machines will be just around the corner. With the 
emergence of artificial intelligence, the works of human art will change deeply. Of course, this may produce a new aesthetic object. Some experts believe that exploratory, heuristic, fuzzy, uncertain and even wrong reasoning methods are more in line with human thinking process [7]. Although artificial intelligence can bring various conveniences in many aspects, generally speaking, the design of environment is not only a technical work, but also the result of the comprehensive design of human emotions. When discussing artificial intelligence, people should pay attention to that it is difficult for artificial intelligence to express the same reaction with human beings in moral level, aesthetic level and emotional level. When a designer carries out an environmental design task, in addition to the basic design elements, he should also consider factors such as self sensing ability, adaptability and individual emotional differences. These need the participation of designers, and they need to understand and respect the demands of owners through repeated exchanges and communication with owners. Although, in big data analysis, artificial intelligence may obtain various comprehensive data for the demands of owners, and can refer to the set template to carry out a variety of analysis, it is difficult for artificial intelligence to make an appropriate response on the subtle level of morality, aesthetics and emotion. Good and bad are easy to judge, but like and dislike, beauty or not, because of individual differences and there is a huge gap. Therefore, the application of artificial intelligence in environmental design, whether at present or in the future, is difficult to completely replace the unique creativity of designers. Although people will lag behind artificial intelligence in the editing ability of hardware or software, in the emotional, aesthetic and moral aspects, human beings will always be the master of design. There is no doubt about this, both now and in the future. When artists talk about the topic of science and art, they often separate them and attribute the problem to how to express them. The rise of artificial intelligence makes this problem extremely complex. Artificial intelligence transforms the problem of creative expression into the problem of legitimacy of creative subject. Can AI have the ability of independent creation and design? Can human creative behavior and artificial intelligence creative behavior be integrated $[8,9]$ ? Many scholars have discussed the influence of artificial intelligence on artistic creation, and pointed out that the artistic creation of artificial intelligence is only a non artistic "pure machine and technology activity" [10-12]. However, people should be aware that in a wide range of civil design, the application of artificial intelligence may greatly meet a wide range of design needs. In a large number of design activities, personalized creation sometimes does not meet the functional requirements to the greatest extent. The objective and rational analysis of big data by artificial intelligence may make it significantly ahead of human designers in meeting the functionality and customer satisfaction. In a sense, artificial intelligence is the intermediate of the demand confrontation between Party A and Party B. It can understand customers and meet their needs to a great extent, and the creativity of design activities has evolved into accessories to meet the needs of
Party A. For future designers, full cooperation with AI is the only choice for design activities.

\section{Conclusion}

The reason why design is called art is because of the humanistic value contained in its design activities. In other words, the essence of art is to create "poetic" truth "and" meditation "rather than" calculation ". The value goal of design activities is to meet the spiritual demands of human beings, and the design works with humanistic value will be recognized as works of art. Although the trend of de decoration in modern times seems to be a negation of design activities, especially decoration activities, in fact, the design concept of "less is more" is just the essence of contemporary human artistic spiritual activities. From this point of view, through the use of artificial intelligence, future designers can greatly simplify the basic work in the current design activities and focus on creative design. Designers can jump out of the occupation limit and reduce the accumulation of basic knowledge, and directly engage in "poetic" pure creative activities. In today's economic life, the creation of value has shifted from goods to service and experience, even personalized experience. In the process of this transformation, compared with the past, technological innovation is particularly rapid. Artificial intelligence, machine learning and augmented reality are driving great changes. In such a constantly changing process of economic development, designers' ability and way of thinking is becoming a crucial solution node for future business success. Although artificial intelligence may make the work of modern designers obsolete, the development of artificial intelligence will enable designers to redesign and create their design documents. The boundary between designers and non designers will be more and more blurred. The key lies in the cooperation and co creation between human and artificial intelligence to find a balance between technology, design thinking and design. Perhaps in the near future, the designer industry will be redefined.

\section{References}

1. SU D. Lost and known way back: a glimpse of the history of ChinaYs environmental art development[M]. Beijing: China Construction Industry Press, 2014.

2. Wang Q, Yu DF, Zhang W P. Research on the preliminary teaching model of environmental design: Taking Ohio State University as an Example[J]. China Architecture Education, 2016,4: 78-82.

3. Park L, Kyung A, Lee H. A Study on the design meaning development by process of analogical design thinking - focusing on the application in apartment interior design prototype[J]. Journal of the Korean Institute of Interior Design, 2006, 15(5):77-84.

4. Bjoegvinsson E, Ehn P, Hillgren P A. Design things and design thinking: Contemporary participatory design challenges[J]. Design Issues, 2012, 28(3):101116. 
5. Li S, Hou Y . Study on the architecture design and interior decoration based on VR technology and computer simulation platform[J]. Paper Asia, 2019, 35(2):54-57.

6. Menezes J. From tinkering methods to design thinking: primordial thoughts in design research $[\mathrm{J}]$. Proceedings of the Design Society International Conference on Engineering Design, 2019, 1(1): 39113918.

7. Shi CY. Principles of Artificial Intelligence[M]. Beijing: Tsinghua University Press, 1993.

8. Lindsay Tan M.F.A. A Review of environmental symbology: origins and contributions toward a theoretical framework $[\mathrm{J}]$. Journal of Interior Design, 2011, 36(2) : 39-49.

9. uh Juneho. The archi-semiotic characteristics of spatial modality in interactive space - focus on gilbert simondon's information of technology[J]. Journal of the Korean Institute of Interior Design, 2013, 22(1):75-84.

10. Wang W, Huang Y. Synergy, Value and singularity: art reflection under the challenge of artificial intelligence[J]. Art Academia, 2018, 2: 4-8.

11. Susanna L. Being with technique-technique as beingwith: The technological communities of gilbert simondon[J]. Continental Philosophy Review, 2019, 52(3) : 299-310.

12. Sun $\mathrm{W}$, Gao $\mathrm{Y}$. The design of university physical education management framework based on edge computing and data analysis[J]. Wireless Communications and Mobile Computing, 2021, 2: 18. 